\title{
The Network Transparency Concept in Fieldbus Based Distributed Systems
}

\author{
Pedro F. Rosa ${ }^{\dagger}$, Francisco Vasques $\$$ and Robert Valette \\ \{rosa; vasques; robert\}@laas.fr \\ LAAS du CNRS \\ 7, Avenue du Colonel Roche \\ 31077 Toulouse France
}

\begin{abstract}
In this paper, we are interested in the study and analysis of how activities are executed at the lowest level of the manufacturing networks hierarchy. At this level, a Real-Time Distributed Database (RTDD) provides a logical link between local applications. The role of the Fieldbus network is to link local sites enabling their interactions and preserving RTDD temporal and spatial consistency.

Specific characteristics of Fieldbus supported RTDDs, such as a "deterministic transaction subset", allow us to introduce the "network transparency" concept, which is the basis of a proposed overall system development.

An [Iso, SC5] based model is presented, providing an efficient method to describe the network transparency concept into standardised description techniques.
\end{abstract}

\section{1 - Introduction}

\section{Manufacturing systems hierarchy}

A Computer Integrated Manufacturing (CIM) system requires an organisational structure that supports a wide variety of activities. These various different activities have been divided in four different functional areas [McG, 88]:

- Business data processing tasks;

- Computer aided design (CAD) tasks;

- Computer aided manufacturing (CAM) tasks;

- Flexible manufacturing systems (FMS) tasks

This organisational structure is standardised by ISO Reference Model for factory automation [Iso, SC5], which defines a 6 level hierarchy supporting all functional areas, from enterprise down to equipment level.

At each hierarchy level, the model assigns a list of activities to be done. This implies that there exists an underlying computing structure which supports all

\footnotetext{
$\dagger$ Mr Rosa, supported in part by a CNPq grant, is also affiliated to LSI EPUSP, Brazil, and can be contacted via e-mail as frosi@1si.usp.br

\$ Mr Vasques, supported in part by a JNICT grant, is also affiliated to FEUP, Portugal and can be contacted via e-mail as vasques@fe.up.pt
}

hierarchy levels. This computational structure, where needs are different in nature and geographically dispersed, must be organised in a distributed and hierarchical scheme. This hierarchy is built according to time constraints, which each network is expected to support [Ple, 88] [Ull, 91].

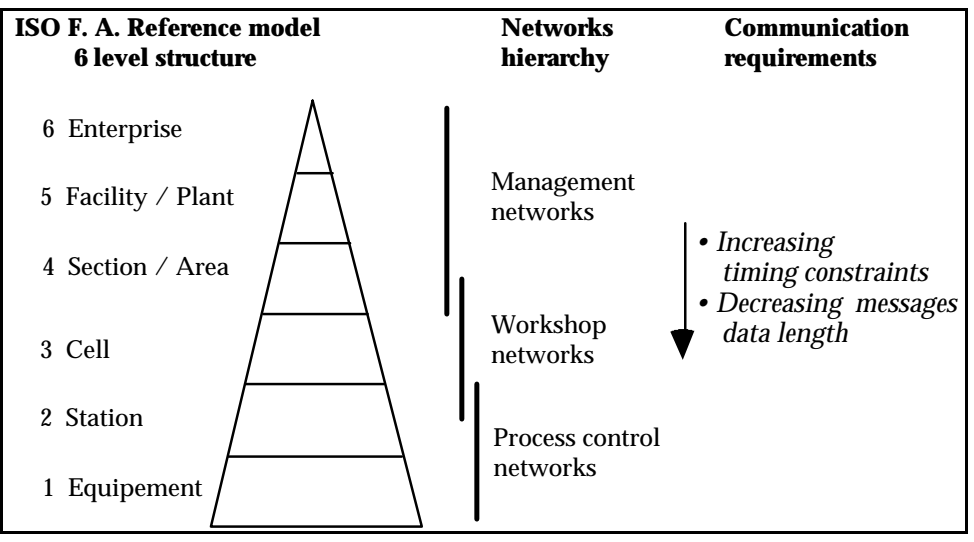

ISO Factory Automation Reference Model

\section{Process control networks: Fieldbuses}

The primary purpose of the lower level of automation is to manage the process as perceived through the sensors and to act upon it through actuators. At this level, automation devices control individual process variables in such a way that the environment achieves a pre-defined manufacturing profile. Units at station level (level 2) must provide level 1 automation devices with such a manufacturing profile, under the form of set-point data or process variable bounding limits. Fieldbus networks are intended to ensure communication between devices within these 2 lower levels.

\section{Data timing characteristics}

At these 2 lower levels, individual applications running at local sites cannot be considered as isolated ones. They are generally components of a global distributed application. The role of the network is to link local sites enabling their interaction. A Real-Time Distributed Database (RTDD) provides the logical link between individual applications and thereby guarantees the correct evolution of the global application. 


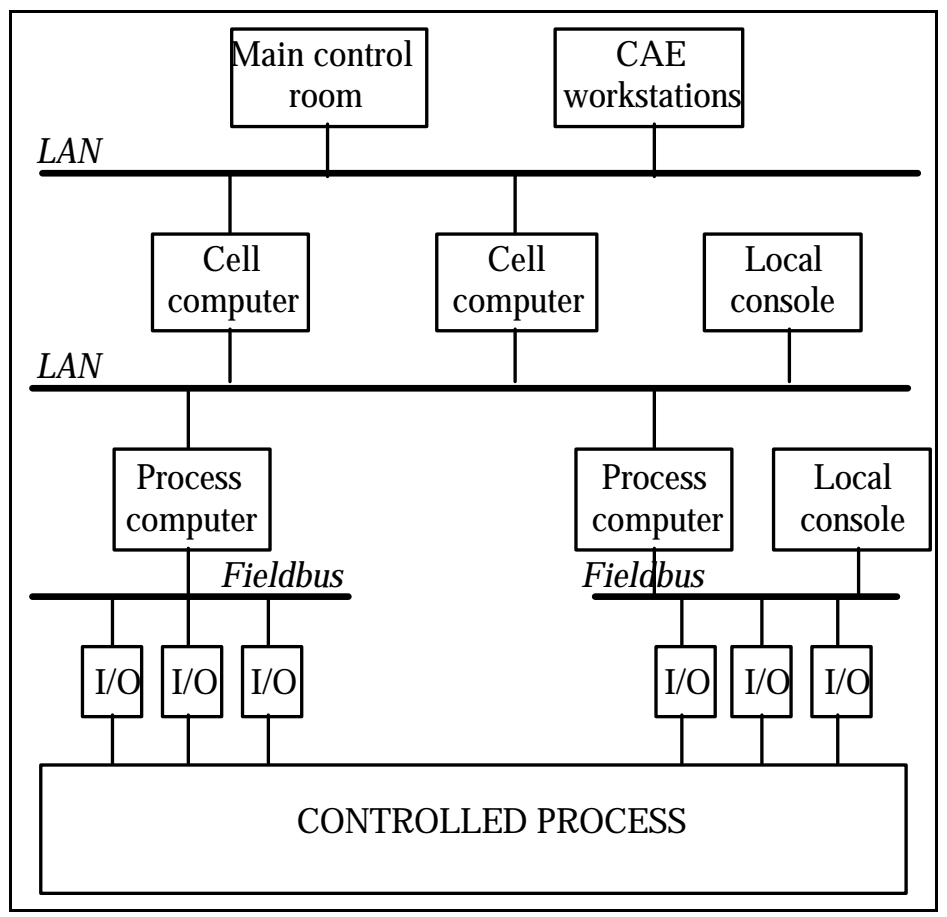

Architecture of a distributed controlling system

In typical real-time applications, the computer system must execute a wide range of operations in parallel. In distributed computing systems, this operations are allocated to different sites. In order to guarantee a consistent behaviour of the distributed system, at each time, each node must perceive the environment as in the same way as the other nodes. Such consistency requirements of data objects may be stated as:

- Temporal consistency: whichever the RTDD object, its stored value must always be representative of the related real-world entity. This means that as the environment evolves, RTDD object values must be periodically updated at convenient rates, in order to provide the correct environment perception to the computer system.

- Spatial consistency: different distributed copies of the same original RTDD object must always store the same value, in order to provide the computer system with a consistent view of the external system. This means that atomicity in multiple object value updating must be guaranteed.

The Fieldbus network must meet data temporal and spatial consistency constraints in order to support the desired RTDD. Temporal consistency is often ensured either by extended use of time stamps, or by validity status, while spatial consistency relies on the definition of the distributed system global state.

\section{2: Real-Time Distributed Databases}

\section{1: Introduction}

Distributed databases in which transactions have timing constraints, such as deadlines, are called real-time distributed databases (RTDD). RTDDs are becoming increasingly important in a wide range of manufacturing facilities, more specifically when the real-time global application can be seen as a distributed set of controlling and controlled processes (the later often referred as environment). In these kind of applications, the correctness of the system not only depend on logical results, but also on time within which results are produced. The RTDD, which provides the logical link for the global real-time application, must schedule transactions in such a way that deadlines are always satisfied, and thus data requirements satisfied.

In RTDDs it is useful to classify transactions as hard and soft real-time transactions. Hard real-time transactions are defined as those transactions whose timing constraints must be guaranteed. Missing deadlines of this type of transaction may result in catastrophic consequences. In contrast, although soft real-time transactions have timing constraints, these constraints may be violated if the situation justifies it. Catastrophic consequences do not result if soft real-time transactions miss their deadlines. Soft real-time transactions are schedule taking into account their timing requirements, but it's not absolutely guaranteed that deadlines will be satisfied.

A Fieldbus based RTDD must support both timecritical and less timing stringent objects, which will be managed, respectively, by hard and soft real-time transactions. This objects may be classified as:

- Hard real-time objects, mainly storing periodic control values and sporadic event notification (and thus storing time-critical values);

- Soft real-time objects storing archival data and desired manufacturing profiles (and thus less stringent timing values).

Objects in the RTDD can be further classified as periodic or aperiodic objects, depending on their value updating characteristics. 


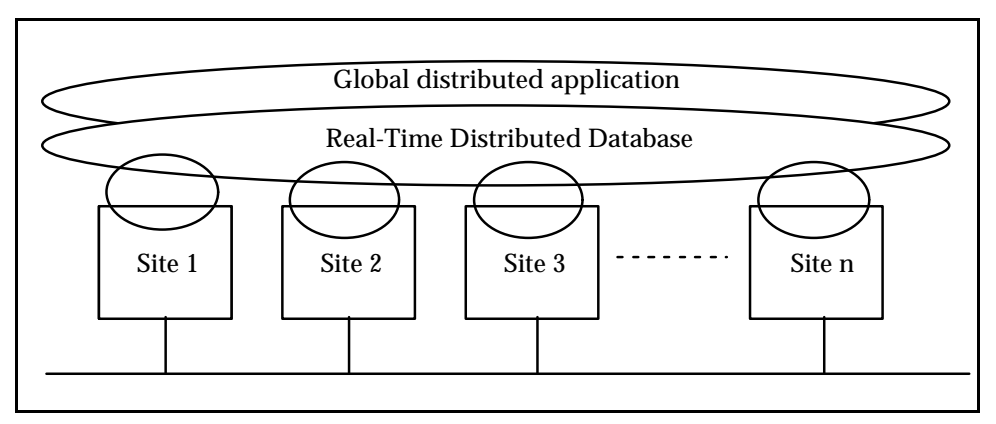

Real-time Distributed Database

\section{2: RTDD triggering mechanisms}

Depending on triggering mechanisms for the transactions to start, two distinctly different approaches to RTDD can be distinguished [Kop, 91]:

- In a purely event-triggered (ET) approach, transactions are initiated whenever a significant state change, i.e. a significant event occurs, spreading the state change within the RTDD. Due to the inherent unpredictability of events and their possible simultaneity, appropriate transaction scheduling algorithms must be employed in order to prevent RTDD timing failures. The definition of event ordering and/or simultaneity must then rely upon object value time stamps, due to the asynchronous nature of the system.

- In a time-triggered (TT) approach, transactions are initiated periodically at predetermined time points, previously matched with the system dynamics. As the dissemination of the environment state is independent of the state changes, the system response delay to an alarm signal is larger in average than in the ET approach. However, the TT approach in the case of real-time systems is usually preferred due to its higher predictability. In this approach, as the control system perceives the environment at pre-defined discrete instants, a precise definition of event order and/or simultaneity is directly obtained.

\section{Network support for ET/TT approaches}

In ET-systems the communication protocols must be event oriented. As only the sender knows when the transaction must be started, all the communication is under the sender initiative. Explicit flow control mechanisms must be employed in order to avoid the network congestion in the case of simultaneous events. The temporal uncertainty [Kop, 91] of asynchronous protocols, i.e., the protocol latency jitter, can be significant and has an adverse effect on the temporal accuracy of the information.

In TT-systems the communication protocols must be state oriented. Each message corresponds to a state dissemination as the result of a periodic transaction. As
RTDD transactions are staticly defined, the transmission initiative is no longer of the sender but of the system. We have then a class of network driven transactions, meaning that it's up to the system to take the transaction initiative.

Within the manufacturing networks hierarchy, both approaches can be found at different levels. At the upper levels, due to the communication unpredictability, the ET approach is often preferred. Exceptions to this rule are usually found in systems with high dependability requirements, i.e., systems which must be simultaneously real-time and fault-tolerant. The TT approach is often used at the lower levels (Fieldbus level) leading to cyclic information exchange between automation devices.

\section{3: Related work and main contributions}

During the last few years, the area of RTDDs has been the focus of a serious research effort, because traditional data models and databases are not adequate for timecritical applications.

While theories of concurrency control in conventional database systems and real-time scheduling have both advanced, little attention has been paid to the interaction between concurrency protocols and real-time scheduling algorithms [Sta, 88]. One of the challenges of RTDDs is the emergence of a theory for real-time scheduling and concurrency protocols that maximises both concurrency and resource utilisation, subject to three constraints: data consistency, transaction correctness and transaction deadlines [Sta, 88].A general RTDDs survey can be both found in [Aud, 92] and [Ram, 93].

It's our belief that Fieldbus supported RTDD must be mainly based on the TT approach, due to the inherent periodic nature of the supported activities and the resulting transaction timing predictability. Moreover, we believe that, whichever the RTDD modelling approach, the fundamental issue of transaction management is that it must directly incorporate the controlled system real-time characteristics, such as periodicity, data validity intervals, maximum admissible jitter, etc.

\section{Client-Server model}

The conventional solution of database (non timecritical) problem is usually based on the client-server paradigm, where data consistency is ensured by the extended use of locking mechanisms. In this model, the transaction initiative is up to the client, which demands a service (the transaction) to the server. The transaction is considered completed when the client has finished all the related operations. 
Directly using the client-server model in a real-time database is not possible because low priority transactions can and will block higher priority transactions, leading to timing requirement failures.

To solve this problem, new techniques are necessary to manage database consistency. They must be compatible with time-driven scheduling. Meeting response-time requirements is possible if a subset of serialisable schedules is found, which avoids unpredictable priority inversions. Intuitively, avoiding unbounded transaction blocking, one guarantees execution time predictability and consequently it's possible to solve the temporal data consistency problem.

This problem has been recently analysed by several authors (see [Aud, 92] and [Ram, 93] for references) and its solutions are specially suited for event-triggered (ET) database approaches.

\section{TT based model}

Time-triggered (TT) based RTDDs are based on the off-line scheduling of performed activities, generating a set of time-lines, one for each operational mode of the system. These off-line schedules must consider all object dependencies in order to provide an implicit synchronisation at run-time of executed activities.

An example of a mixed TT / ET based RTDD is the FIP supported database [Fip, 92], where periodic and aperiodic transactions coexist in the following way:

- An off-line system scheduling generates an infinite sequence of fixed duration time slots (micro-cycles). Within each micro-cycle, a variable fraction is allocated to execute periodic time-triggered transactions; the remaining fraction may be used for requested aperiodic transactions, mainly event-triggered ones.

\section{3: The network transparency concept}

Within the manufacturing networks hierarchy, timetriggered (TT) and event-triggered (ET) approaches coexist at different levels. At the lower level of this hierarchy, due to the periodic nature of control processes, the TT approach is often employed, in order to increase system predictability.

\section{Fieldbus supported RTDD}

At the lower level of a manufacturing system, the primary purpose of the computing system is to control individual process variables in such a way that the controlled process follows a pre-defined trajectory.

At this level, executed activities are essentially of periodic and time constrained nature [Ple, 88], thus RTDD objects are mainly periodic hard real-time objects, related to process control activities. As well, an important subset of objects are the aperiodic hard real-time objects, which are mainly related to exceptional events such as alarms.

In order to provide an efficient time-critical data management, i.e., preserving data temporal and spatial consistencies, the Fieldbus supported RTDD must comply with the following features:

- As an important object subset (the periodic hard realtime subset) is by nature staticly pre-defined, in order to increase overall predictability, the system must provide means to the off-line scheduling of related transactions. Due to this pre-defined transactions subset, respective deadlines may be off-line guaranteed, leading to a lower overall system run-time complexity.

- As one RTDD object value usually has several simultaneous consumers, guaranteeing RTDD data spatial consistency is a fundamental issue. Solutions to this problem are usually based on the use of data multicasting mechanisms and on the definition and verification of system global state.

\section{The network transparency concept}

In Fieldbus supported RTDDs, as TT transactions are off-line scheduled, such activities may be executed under the network control. As a consequence, global application activities are not burdened by the task of ensuring the spatial consistency.

The RTDD data spatial consistency is ensured in a three-step mode: first, a local application task updates the local object value and lock it against reading operations; second, a TT transaction executed under the network control, ensures the object spatial consistency (during the TT transaction execution all object values, original one and copies remain locally locked against reading operations). Then, original value and copies are simultaneously unlocked and become available for local reading operations of global activities.

This modular structure of database management, avoids mechanisms such as distant table lock-up and real-time data synchronisation conflicts, which are one of the major unpredictability sources in client-server databases.

The network transparency concept is a consequence of decoupling between updating local and distant object values. As a consequence, higher degree of system predictability can be achieved, mainly because synchronisation problems are encapsulated within local sites and are transparent at global level. 


\section{4: Modelling the network transparency concept}

The ISO Reference Model for factory automation defines an abstract model for distributed application description [Iso, SC5].

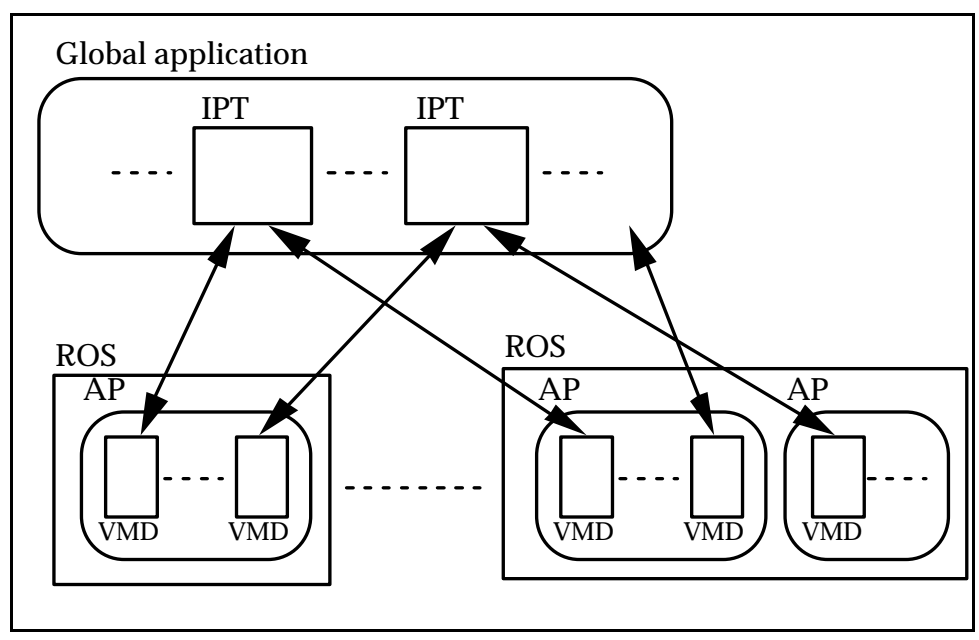

Distributed application description

Basically, this abstract model states that:

- The global application is broken down into basic activities called Information Processing Tasks (IPTs). Such tasks may be, for instance, distributed application controlling / monitoring tasks.

- Each IPT is executed using a set of virtual distributed resources called Virtual Manufacturing Devices (VMDs);

- At each distributed system node, the set of available resources (hardware, software, etc.) is called Real Open System (ROS);

- The ROS is subdivided into one or several Application Processes (APs), which represent physical processes. Each AP executes a set of one or more VMDs;

- Each VMD contains a set of Domains (IPT pictures), one optional Virtual Filestore (VF), and a set of communication capabilities called Application Entity (AE);

- Each AE has its respective Application Program Interface (API).

\section{Distributed application specification}

The RTDD which supports the global distributed application is locally based on the individual VMD databases: the VF. At each one VMD, the VF allows the storage of system variables, which will be exchanged through the network (database transaction) and used by the respective IPTs.

A fundamental difference between a Fieldbus supported RTDD and a traditional network based one (client-server model) is here reminded:
- Client-server RTDDs are based on ET transactions which are transactions executed as consequence of IPT explicit requests: application synchronised transactions.

- Fieldbus RTDDs based on TT transactions for periodic activities, leaves such type of transactions to the system control, i.e., it's up to the network to start transactions at the previously defined instants: network driven transactions.

As a consequence, in Fieldbus based RTDDs, it is up to the network to assure data temporal consistency. The related domains limit themselves to store/recall variables in the VMD VF, assuming that the underlying network will execute the desired transaction (implicit request). This network transparency concept leads to a modular RTDD modelling technique, reducing the overall problem complexity.

\section{5: Concluding remarks}

In this paper, we have been concerned by the study and analysis of the activity executions within the context of Real-Time Distributed Database (RTDD) based on a Fieldbus.

Specific characteristics of Fieldbus supported RTDDs, such as a pre-defined transaction subset, allow us to introduce the network transparency concept, which is the basis of a proposed overall system development.

In a Fieldbus RTDD, the global application may be seen as a set of local applications which limit themselves to local operation. The global database consistency problem is assumed to be guaranteed by the underlying network, which executes desired transactions at the pre-defined instants (implicit request). This network transparency concept leads to a modular RTDD modelling technique, reducing the overall problem complexity.

An [Iso, SC5] based model is presented, providing a method to describe the network transparency concept based on standardised description techniques. This means system specification through abstract objects (ROS, AP, VMD, IPT, API, ...), as well as object interaction via VF. As far as we know, the use of this reference model to highlight this network transparency concept has never been done before.

\section{References}

[Aud, 92]; N. Audsley, A. Burns, M. Richardson, A. Wellings; "Data Consistency in Hard Real-Time Systems" YCS203, Dept. of Computer Science, University of York 
[Dec, 93]; J. Decotignie, P. Raja; "Fulfilling Temporal Constraints in Fieldbus"; in Proc. of IECON'93, pp 519524

[Fip, 92]; "The Fip Protocol", P. Leterrier, Club Fip

[Iso, SC5]; "A Reference Model for Discrete Parts Manufacturing"; ISO TC184/SC5/WG1-N58, ISO

[Kop, 91]; H. Kopetz; "Event-Triggered versus Time-Triggered Real-Time Systems"; in Operating Systems of the 90's and Beyond, LNCS 563, Springer Verlag 1991

[McG, 88]; "MAP/TOP in CIM Distributed Computing"; L. McGuffin, L. Reid, S. Sparks; IEEE Network, vol. 2 n³, May 1988

[Pim, 90]; J. Pimentel; "Communication Networks for Manufacturing"; Prentice Hall, 1990

[Ple, 88]; Pleinevaux, J. Decotignie; "Time Critical Communication Networks"; IEEE Network, vol. 2 n³, May 1988

[Ram, 93]; K. Ramamritham; "Real-Time Databases"; Journal of Distributed and Parallel Databases; vol. 1 (1993), pp 199-226

[Sta, 88]; J. Stankovic, K. Ramamritham; "Hard Real-Time Systems"; IEEE Computer Society Press, 1988

[Tho, 89]; J. Thomesse, T. Lainé; "The Fieldbus Application Services"; in Proc. of IECON'89, pp 526-530

[Ull, 91]; G. Ulloa; "Fieldbus Application Layer and Real-Time Distributed Systems"; in Proc. of IECON'91; pp 16791683 